\title{
PROBABILITY AND INTERPOLATION
}

BY

\author{
G. G. LORENTZ ${ }^{1}$ AND R. A. LORENTZ
}

\begin{abstract}
An $m \times n$ matrix $E$ with $n$ ones and $(m-1) n$ zeros, which satisfies the Pólya condition, may be regular and singular for Birkhoff interpolation. We prove that for random distributed ones, $E$ is singular with probability that converges to one if $m, n \rightarrow \infty$. Previously, this was known only if $m>(1+\delta) n / \log n$. For constant $m$ and $n \rightarrow \infty$, the probability is asymptotically at least $\frac{1}{2}$.
\end{abstract}

1. Introduction. For an $m \times n$ matrix $E=\left(e_{i k}\right)_{i=1}^{m}{ }_{k=1}^{n}$ with elements that are zeros and ones, and with exactly $n$ ones, we study the Birkhoff interpolation problem: $P^{(k-1)}\left(x_{i}\right)=c_{i k}$ if $e_{i k}=1$, for polynomials $P$ of degree $<n-1$. If the problem is solvable for all real knots $X: x_{1}<\cdots<x_{m}$ and all data $c_{i k}$, the matrix $E$ is regular (or poised). Otherwise it is singular. Schoenberg [13] has asked to determine the character of a matrix by using only the distribution of ones in it. This problem proved to be extremely difficult, and one is satisfied to answer some particular questions in this direction. For an exposition of known results in Birkhoff interpolation see [8], [9]. We shall use notations and results of these two papers.

The main result of this paper is that for large $m$ and $n$, a Pólya $m \times n$ matrix $E$, with $n$ ones distributed at random, is singular with probability close to 1 if $m$ and $n$ are large (Theorem 1 below). Previously, this result was known [10] under the assumption that

$$
m \geqslant(1+\delta) \frac{n}{\log n}, \text { for any constant } \delta>0 .
$$

A few words of justification for our Theorem 1, which is somewhere between the result of [10] and the ultimate truth.

Probability theorems of the kind treated here, depend, of course, upon criteria of regularity and singularity of matrices. At present, there are only four such criteria of real importance. We list them below, indicating the probability that they apply to an $m \times n$ Pólya matrix $E$ (these probabilities are either easy to find, or follow along the lines of the present paper).

(1) The only general test of regularity is given by the theorem of AtkinsonSharma [1] which in hidden form is present already in G. D. Birkhoff's paper [2].

Received by the editors April 30, 1980 and, in revised form, March 12, 1981.

1980 Mathematics Subject Classification. Primary 41A05; Secondary 60C05, 60E99.

Key words and phrases. Birkhoff interpolation, Pólya matrix, regularity and singularity, coalescence of rows, probability of singularity, hypergeometric distribution.

${ }^{1}$ Supported by NSF Grant MCS-7904689. 
Probability of its applicability tends to zero for $n \rightarrow \infty$. The problem to improve this theorem is well known, but no progress has been achieved.

The following are three (quite different) tests of singularity.

(2a) A matrix $E$ is singular (Lorentz [7]) if it has odd supported sequences and if all but one of its rows contain exactly one one. Probability of applicability tends to 0 for $n \rightarrow \infty$.

(2b) A matrix $E$ is singular (Lorentz-Zeller [11]) if it has a row consisting of a supported one. A stronger (and more difficult to prove) version (Lorentz [6]) replaces this by an arbitrary row which contains exactly one odd supported sequence. Probability of applicability tends to 1 , but only if $m$ is large (hence condition (1.1)).

(2c) A matrix $E$ is singular if $\Delta \equiv 1(\bmod 2)$, where $\Delta$ is some integer easily computed if $E$ is given (Lorentz [8]); for three row matrices only, this is equivalent to a test of Karlin and Karon [4]. The probability that the test applies converges to $\frac{1}{2}$ as $n \rightarrow \infty$.

To improve our Theorem 1, one has either to find a criterion of regularity which has probability $\geqslant c_{0}>0$, or to find a singularity test which is applicable with probability converging to one. One of the points of this paper is perhaps that it underlines problems, whose solution would signify an important achievement in Birkhoff interpolation.

Necessary for the regularity of $E$ is the forward Pólya condition, which means that for $k=1, \ldots, n$, the first $k$ columns of $E$ contain at least $k$ ones. Then $E$ is called a Pólya matrix. Equivalent to this is the backward Pólya condition: any last $n-k$ columns of $E$ contain at most $n-k$ ones. Both forms of the Pólya condition are equivalent, if $E$ has exactly $|E|=n$ ones. If $|E|<n$, one should use the backward Pólya conditions, and if $|E|>n$, the forward condition.

Let $M(m, n)$ be the set of all $m \times n$ matrices $E$ with $n$ ones, let $P(m, n)$ be its subset consisting of all Pólya matrices. We allow empty rows (without ones) in $E$, but will always assume $m \leqslant n$. For these matrices we have the counts [6]

$$
\begin{gathered}
|M(m, n)|=\left(\begin{array}{c}
m n \\
n
\end{array}\right), \\
|P(m, n)|=\frac{1}{(n+1)}\left(\begin{array}{c}
m(n+1) \\
n
\end{array}\right) \leqslant \text { Const } \frac{1}{n}|M(m, n)| .
\end{gathered}
$$

This inequality shows that most $E \in M(m, n)$ are singular. The purpose of the present paper is to show that Pólya matrices with large $m, n$ are singular with probability close to one. We shall establish (see announcement [12])

THEOREM 1. For each $\varepsilon>0$ there is an $n_{0}$ with the property that for $m \geqslant n_{0}$, all but $\varepsilon|P(m, n)|$ matrices of the class $P(m, n)$ are singular.

Theorem 2. Let $\varepsilon>0$ and $m \geqslant 3$ be given, let $p=[m / 3]$. Then for $n \geqslant n_{0}$ the number of regular matrices of the class $P(m, n)$ does not exceed

$$
\left(2^{-p}+\varepsilon\right)|P(m, n)| \text {. }
$$

First we state the test of singularity that we shall need. 
A row $F$ of zeros and ones from matrix $E$ can be also represented by the positions of ones in $F$. For example to $F_{1}=(1010100100)$ corresponds $\tilde{F}_{1}=$ $(1,3,5,8)$. Precoalescence of $F_{1}$ with respect to another row $F_{2}$ (see [9, p. 202]), denoted by $\left(F_{1}\right)_{2}$, leads to precoalescnece $\left(\tilde{F}_{1}\right)_{2}$. For example, if $F_{2}=(1001100110)$, then $\tilde{F}_{2}=(1,4,5,8,9)$ and $\left(F_{1}\right)_{2}=(0110010001),\left(\tilde{F}_{1}\right)_{2}=(2,3,6,10)$. There is a corresponding operation for permutations of $\tilde{F}_{1}$, thus $(3,5,8,1)_{2}=(3,6,10,2)$. We shall write $\left(\tilde{F}_{1}\right)_{23}$ for the sequence $\tilde{F}_{1}$ precoalesced to the coalescence of rows $F_{2}$ and $F_{3}$, and $\tilde{F} \tilde{F}^{\prime}$ for the sequence of elements of $\tilde{F}$ followed by those of $\tilde{F}^{\prime}$.

The following obvious remark about precoalescences of two permutations of row $\tilde{F}_{1}$ with respect to a fixed row $\tilde{F}_{2}$ will be used in $\$ 3$.

REMARK. If in the row $\tilde{F}_{1}$, positions of two integers $l, \bar{l}$ are interchanged, then also in $\left(\tilde{F}_{1}\right)_{2}$ exactly two integers are interchanged, namely images of $l, \bar{l}$ under precoalescence.

The singularity theorem mentioned above can be explained as follows. Let $E_{0}$ be any $m_{1} \times n$ matrix of zeros and ones with $m_{1} \leqslant m$. We shall say that $E_{0}$ is a singular strip, if any matrix $E \in M(m, n)$ containing the rows of $E_{0}$ as consecutive rows, is singular. As a special case of a theorem in $[8$, p. 188] we have:

Proposition 1. Let $E_{0}$ be a $3 \times n$ matrix consisting of the rows $F_{1}, F_{2}, F_{3}$. The two sequences

$$
\begin{aligned}
S & =\left(\left(\tilde{F}_{1}\right)_{2} F_{2}\right)_{3}, \\
S^{\prime} & =\left(\tilde{F}_{1}\right)_{23}\left(\tilde{F}_{2}\right)_{3}
\end{aligned}
$$

consist of the same integers (namely, of the positions of ones in the coalescence of $E_{0}$ to one row less the ones in $\left.F_{3}\right)$. Let $\Delta=+1($ or $\Delta=-1)$ if $S$ requires an even (or an odd) number of permutations in order to bring it to the natural order, let $\Delta^{\prime}$ be a similar number for $S^{\prime}$. If

$$
\Delta \neq \Delta^{\prime}
$$

then $E_{0}$ is a singular strip.

This statement remains true if $E_{0}$ is formed by three groups of rows $E_{1}, E_{2}, E_{3}$ (instead of three rows), but then for $i=1,2,3, F_{i}$ should mean the coalescence of the mattrix $E_{i}$ to one row.

2. Probabilities: hypergeometric distribution. Let $G$ be a set of cells, containing $N=|G|$ of them, among the cells we distribute $n \leqslant N$ ones. Each cell can accept only one one. Let $G_{1} \subset G$ be a subset of $G$ consisting of $N_{1}$ cells. Among the $\left(\begin{array}{l}N \\ n\end{array}\right)$ possible distributions of ones we single out those which have $k$ ones in $G_{1}$ (and $n-k$ ones in the complement of $G_{1}$ ). The number of all distributions with this property will be

$$
\left(\begin{array}{c}
N_{1} \\
k
\end{array}\right)\left(\begin{array}{c}
N-N_{1} \\
n-k
\end{array}\right) .
$$

In other words, the probability of $k$ ones falling into $G_{1}$ is

$$
p_{N, N_{1}, n}(k)=p_{n}(k)=\left(\begin{array}{c}
N_{1} \\
k
\end{array}\right)\left(\begin{array}{c}
N-N_{1} \\
n-k
\end{array}\right) /\left(\begin{array}{c}
N \\
n
\end{array}\right) \text {. }
$$


We are interested in the cases when $k$ is far away from $n N_{1} / N$. By means of Stirling's formula and some elementary computations one gets

LEMMA 1. For $n \rightarrow \infty$ and each $\varepsilon>0$,

$$
\sum_{\left|k-n N_{1} / N\right|>e n} p_{n}(k) \leqslant \rho^{n},
$$

for some $\rho, 0<\rho<1$, dependent on $\varepsilon$.

Actually, stronger estimates are known in probability theory. Let $X_{1}$ be the random number of ones falling into $G_{1}$. The random variable $X_{1}$ has expectation $E\left(X_{1}\right)=n N_{1} / N$ and the hypergeometric distribution

$$
P\left(X_{1}=k\right)=\left(\begin{array}{c}
N_{1} \\
k
\end{array}\right)\left(\begin{array}{c}
N-N_{1} \\
n-k
\end{array}\right) /\left(\begin{array}{l}
N \\
n
\end{array}\right) \text {. }
$$

LEMMA $1^{*}$. One has

$$
P\left(\left|X_{1}-\frac{n N_{1}}{N}\right| \geqslant \varepsilon n\right)<2 \exp \left(-\frac{1}{2} n \varepsilon^{2}\right)
$$

For the proof see for example Serfling [14, p. 41, Corollary 1.1]. A simple proof can be also read off formulas (4.8)-(4.10) in Kemperman [5].

If $G$ is an $m \times n$ table, then a distribution of $n$ ones in $G$ can be interpreted as a matrix $E \in M(m, n)$. Often we shall have a fixed number $p$ of subsets $G_{q}$ of $G$ (for example, some rows of $G$ ) with union $G$. Then a matrix $E$ will define submatrices $E_{q}, q=1, \ldots, p$, contained in $E_{q}$. The inequality (2.2) means that for each $q$, all but $\rho^{n}\left(\begin{array}{l}N \\ n\end{array}\right)$ matrices $E$ will have in $E_{q}$ a number of ones satisfying

$$
\left|k_{q}-N_{q} / m\right| \leqslant \varepsilon n \text {. }
$$

If we want (2.5) to be valid for each $q=1, \ldots, p$, we must exclude a larger set of size $p \rho^{n}\left(\begin{array}{l}N \\ n\end{array}\right) \leqslant \rho_{1}^{n}\left(\begin{array}{c}N \\ n\end{array}\right), 0<\rho_{1}<1$. We have from Lemma 1:

LeMMA 2. All matrices $E \in M(m, n)$, except for at most $\rho^{n}|M(m, n)|$ of them, have in $E_{q}, q=1, \ldots, p$, a number $k_{q}$ of ones which satisfies (2.5).

We shall say that some phenomenon happens for "almost all" matrices of a class $\mathcal{E}_{n}$, if for some $\rho, 0<\rho<1$ and all large $n$ it happens for at most $\rho^{n}\left|\mathcal{E}_{n}\right|$ matrices of $\mathcal{E}_{n}$. From (1.1) it follows: if some phenomenon happens for "almost all" matrices of the class $M(m, n)$, then the same is true for matrices of the class $P(m, n)$.

3. Properties of matrices. We collect here some lemmas needed for our theorems. Let $P(m, n)$ and $P(m, n ; n+q), q=1,2, \ldots$, respectively be the numbers of $m \times n$ matrices which satisfy the forward Pólya condition and have $n$, respectively $n+q$ ones.

Proposition 2. With a constant $C$ depending only on $q$,

$$
\begin{aligned}
P(m, n+q) & \leqslant C m^{q} P(m, n), \\
P(m, n ; n+q) & \leqslant C m^{q} P(m, n) .
\end{aligned}
$$


Proof. Using (1.1) we have

$$
\begin{aligned}
P(m, n+1)= & \frac{1}{n+2}\left(\begin{array}{c}
m n+2 m \\
n+1
\end{array}\right) \\
= & \frac{1}{(n+1)(n+2)} \frac{(m n+m) \cdots(m n+m-n+1)}{n !} \\
& \times \frac{(m n+2 m) \cdots(m n+m+1)}{(m n+2 m-n-1) \cdots(m n+m-n+1)} \\
\leqslant & \frac{1}{(n+1)(n+2)}(n+1) P(m, n)(m n+2 m)\left(1+\frac{1}{m-1}\right)^{m-1} \\
\leqslant & e m P(m, n) .
\end{aligned}
$$

By induction, (3.1) follows with $C=e^{q}$; and (3.2) follows from

$$
P(m, n ; n+q) \leqslant P(m, n+q) \text {. }
$$

A column $k, k=2, \ldots, n$ in an $m \times n$ matrix $E$ will be called special, if the submatrix of $E$ consisting of the first $k-1$ columns satisfies the backward Pólya condition. If $E$ has $N<n$ ones, special columns of $E$ exist:

Proposition 3. If $E$ has $N<n$ ones, then there are at most $N$ positions $k$ that are not special.

Proof. Let $m_{k}, k=1, \ldots, n$, be the Pólya function of $E$, namely the number of ones in the $k$ th column of $E$. We want to prove that for at least $n-N$ values of $j$,

$$
\sum_{l=i}^{j} m_{l}<j-i+1, \quad i=1,2, \ldots, j-1 .
$$

We will consider the Pólya numbers as having subscript modulo $n$ :

$$
m_{k+l n}=m_{k}, \quad l=0, \pm 1, \ldots
$$

An interval $\left[i_{0}, j_{0}\right]$ with $-\infty<i_{0} \leqslant j_{0}<\infty$ will be called a chain if

$$
\sum_{l=i_{0}}^{j} m_{l} \geqslant j-i_{0}+1 \text { for all } j \text { with } i_{0} \leqslant j \leqslant j_{0} \text {. }
$$

Of particular interest are maximal chains, which are not contained in any other chain. It is easy to see that

(a) two different maximal chains are disjoint,

(b) for a maximal chain $[i, j]$, one always has $m_{i-1}=m_{j+1}=0$,

(c) if $m_{i}>0$, then $i$ belongs to some maximal chain.

Thus maximal chains are separated from each other by intervals of zero values of $m_{i}$. Moreover

(d) for a maximal chain $[i, j]$,

$$
\sum_{l=i}^{j} m_{l}=j-i+1 .
$$

It follows from (d) that $N=\sum_{l=1}^{n} m_{l}$, which is equal to the sum of the $m_{l}$ in all the maximal chains, is also equal to the sum of the lengths of all maximal chains. Thus there are exactly $n-N$ integers $j$ in $[1, n]$ which do not belong to any chain. 
To complete the proof, it is now sufficient to observe that an integer $j$ satisfies

$$
\sum_{l=i}^{j} m_{l}<j-i+1
$$

for all $i \leqslant j$ (which implies that $j$ is special), exactly when $j$ does not belong to a chain. Indeed, if $j$ does not satisfy this condition, we take the largest integer $i_{0}$, with $i_{0} \leqslant j$ and

$$
\sum_{l=i_{0}}^{j} m_{l} \geqslant j-i_{0}+1
$$

then for all $j_{1}, i_{0} \leqslant j_{1} \leqslant j$, we must have

$$
\sum_{l=i_{0}}^{j_{1}} m_{l} \geqslant j_{1}-i_{0}+1
$$

This implies that $j$ belongs to the chain $\left[i_{0}, j\right]$.

For three row matrices we need the following singularity theorem, which is of independent interest:

THEOREM 3. Let the $3 \times n$ matrices $E_{1}, E_{2}$ be identical except for their columns $k$ and $k+1$, which are (I) for $E_{1}$ and (II) for $E_{2}$ :

$$
\text { (I): } \left.\left.\quad \begin{array}{rr}
k & k+1 \\
1 & 0 \\
1 & 0 \\
1 & 0
\end{array}\right) ; \quad \text { (II): } \quad \begin{array}{rr}
k & k+1 \\
0 & 1 \\
1 & 0
\end{array}\right) .
$$

Then at least one of the matrices is a singular strip.

Proof. For the matrices $E_{1}, E_{2}$, we compare sequences $S, S^{\prime}$ and numbers $\Delta, \Delta^{\prime}$ of Proposition 1, distinguishing them by subscripts. We want to show that either $\Delta_{1} \neq \Delta_{1}^{\prime}$ or $\Delta_{2} \neq \Delta_{2}^{\prime}$. This will follow from

$$
\Delta_{1} \neq \Delta_{2}, \quad \Delta_{1}^{\prime}=\Delta_{2}^{\prime}
$$

The first row $\tilde{F}_{1}$ of $E_{1}$ and of $E_{2}$ is of the form

$$
\tilde{F}_{1}=\left(l_{1}, \ldots, l_{t}, k, l_{t+2}, \ldots, l_{p}\right) \text {, }
$$

the row $\tilde{F}_{2}$ is almost identical for both matrices, namely $\tilde{F}_{2}=$ $\left(l_{1}^{\prime}, \ldots, l_{s}^{\prime}, k, l_{s+2}^{\prime}, \ldots, l_{q}^{\prime}\right)$ for $E_{1}$ and $\tilde{F}_{2}=\left(l_{1}^{\prime}, \ldots, l_{s}^{\prime}, k+1, l_{s+2}^{\prime}, \ldots, l_{q}^{\prime}\right)$ for $E_{2}$. If we precoalesce $\tilde{F}_{1}$ to $\tilde{F}_{2}$, there will be an overflow $\lambda \geqslant 0$ at $k$, that is, there will be $\lambda$ terms $<k$ in $\tilde{F}_{1}$, which will move to $\lambda$ positions $\geqslant k$ of zeros $z_{q}$ of $F_{2}$. The overflow will move $i_{t-\lambda+1}, \ldots, l_{t}, k$ of $\tilde{F}_{1}$ to $k+1, z_{1}, \ldots, z_{\lambda}$ for the matrix $E_{1}$ and to $k, z_{1}, \ldots, z_{\lambda}$ for $E_{2}$. Terms $<k$ and $>z_{\lambda}$ will be the same in both precoalescences. This shows that

$$
\begin{aligned}
& S_{1}=\left(\bar{l}_{1}, \ldots, \bar{l}_{t-\lambda}, k+1, z_{1}, \ldots, z_{\lambda}, \ldots, l_{1}^{\prime}, \ldots, l_{s-1}^{\prime}, k, l_{s+1}^{\prime}, \ldots, l_{q}^{\prime}\right)_{3}, \\
& S_{2}=\left(\bar{l}_{1}, \ldots, \bar{l}_{t-\lambda}, k, z_{1}, \ldots, z_{\lambda}, \ldots, l_{1}^{\prime}, \ldots, l_{s-1}^{\prime}, k+1, l_{s+1}^{\prime}, \ldots, l_{q}^{\prime}\right)_{3} .
\end{aligned}
$$


Using the remark of $\S 1$, we see that $S_{1}$ is obtainable from $S_{2}$ by an odd number of permutations, and $\Delta_{1} \neq \Delta_{2}$.

We turn our attention to the sequences $S_{1}^{\prime}, S_{2}^{\prime}$. Let $\tilde{F}_{23}$ be the coalescence of rows $\tilde{F}_{2}$ and $\tilde{F}_{3}$. If $F_{2}$ has one in position $k$, we can move it to position $k+1$ before coalescence without changing $\tilde{F}_{23}$. It follows that $\tilde{F}_{23}$ is the same for both matrices $E_{1}, E_{2}$. Also $\left(\tilde{F}_{2}\right)_{3}$ is the same. Hence $S_{1}^{\prime}=S_{2}^{\prime}$ and $\Delta_{1}^{\prime}=\Delta_{2}^{\prime}$.

Theorem 3 is trivial if there is no overflow $\lambda$ in coalescence of $F_{1}, F_{2}, F_{3}$ to one row. In this case, we have to consider the elements of $S, S^{\prime}$ only in the interval $[k, k+2]$. They are

$$
S_{1}=k+2, k+1 ; \quad S_{2}=k+1, k+2 ; \quad S_{1}^{\prime}=S_{2}^{\prime} .
$$

Assumption $\lambda=0$ allows us to extend the statement of Theorem 3 to strips of more than three rows. Desirable pairs of columns $k, k+1$ in $E_{0}$ are of the following two types (I) or (II):

The only nonzero elements of columns $k, k+1$ are contained in the rows $i_{1}<i_{2}<i_{3}$ of $E_{0}$; they form either the submatrix (I) or (II) of (3.6).

Proposition 4. If two $m_{1} \times n$ matrices $E_{1}, E_{2}$ with overflow $\lambda=0$ at $k$ are identical except for their columns $k, k+1$ and are of the types (I) and (II) respectively, then at least one of them is a singular strip.

Proof. After coalescing (without overflow!) the matrices $E_{1}, E_{2}$ to their three rows $i_{1}, i_{2}, i_{3}$, we apply the above argument.

One way to establish that $\lambda=0$ at $k$ for a matrix $E_{0}$ is to show that $k$ is a special position for $E_{0}$. Hence the importance of Proposition 3.

4. Existence of special pairs of columns. For the proof of Theorem 1, we take a fixed integer $p \geqslant 5$ and large $m, m \geqslant 3 p$. We divide the $m$ rows of $E$ into $p$ groups $E_{q}$ of consecutive rows of $E$, each $E_{q}$ consisting of $[m / p]=m_{1}$, rows, plus a remainder of $<m_{1}$ rows, which we will disregard. According to $\S 1$, for "almost all" $E \in P(m, n)$ each $E_{q}$ will have approximately $m_{1} n / m$, more exactly $<(1 / p+\varepsilon) n$ ones. We denote by $\bar{P}(m, n)$ this subset of $P(m, n)$. We shall call two columns $2 k$, $2 k+1$ a special pair for $E_{q}$, if $2 k$ is in special position for $E_{q}$, and if $E_{q}$ is of the type (I) or (II) (of Proposition 4) with respect to the two columns.

PROPOSITION 5. For $p \geqslant 5$, all but at most

$$
C(p) \frac{1}{n} P(m, n)
$$

matrices $E \in \bar{P}(m, n)$ have a special pair in each of the $E_{q}, q=1, \ldots, p$. The constant $C(p)$ depends only on $p$.

Proof. We count the number of $E \in \bar{P}(m, n)$ which have no special pair in one of the submatrices $E_{q}$, for example in $E_{1}$. Let $\varepsilon_{1}$ be this set of matrices. There are at most $(1 / p+\varepsilon) n$ ones in $E_{1}$, hence by Proposition 3 , at most $(1 / p+\varepsilon) n$ columns $2 k$ which are not in special position in $E_{1}$, hence at least $[n / 2]-$ $[(1 / p+\varepsilon) n]$ columns $2 k$ in special position, and at least $[n / 2]-2[(1 / p+\varepsilon) n]$ 
columns $2 k$ with this property and for which columns $2 k, 2 k+1$ contain only zeros. For small $\varepsilon>0$, we obtain $\geqslant c n$ columns for some $c>0$.

In one of these pairs of columns $2 k, 2 k+1$ and some of the rows $i_{1}<i_{2}<i_{3}$ of $E_{1}$ we introduce the group (I) or (II) of ones (see (3.6)). This will transform $E_{1}$ into $\tilde{E}_{1}$ and $E$ into a matrix $\tilde{E} \in P(m, n ; n+3)$. There are at least $c n$ choices of the column $2 k$, at least $(m / p)^{3}$ choices of the rows $i_{1}, i_{2}, i_{3}$. Hence each $E_{1}$ of our type will produce at least Const $m^{3} n / p^{3}$ matrices $\tilde{E}$. Conversely, each matrix $\tilde{E}$ obtained can come only from one $E_{1}$, for $\tilde{E}_{1}$ must have a special pair at the place selected, and can have no other special pair (otherwise this would be a special pair also for $\left.E_{1}\right)$. By (3.2) therefore,

$$
\left|\mathcal{E}_{1}\right| \frac{m^{3} n}{p^{3}} \leqslant \text { Const } P(m, n ; n+3) \leqslant C m^{3} P(m, n),
$$

and we obtain $\left|\mathcal{E}_{1}\right| \leqslant$ Const $P(m, n) / n$.

For the proof of Theorem 2 we need the simpler Proposition 6 below. It is based on the following fact.

Let a certain number $N$ of ones, $C n \leqslant N \leqslant C_{1} n$, where $0<C<C_{1}<1$ be distributed at random into $n$ cells numbered $1,2, \ldots, n$. Then (*) for some $c_{0}>0$, "almost all" distributions contain $\geqslant c_{0}$ groups of cells $2 k, 2 k+1$ with configuration 1,0 , and $\geqslant c_{0} n$ groups with configuration 0,1 .

Consider for example the configuration 1, 0. From Lemma 2 we see that, for "almost all" distributions of ones, of the approximately $n / 2$ positions $2 k$, at least $\left(\frac{1}{2} C-\delta\right) n$ and at most $\left(\frac{1}{2} C_{1}+\delta\right) n$ of them will be occupied by ones. This leaves $\leqslant\left(\frac{1}{2} C_{1}+\delta\right) n$ ones, hence $\geqslant\left(1-\frac{1}{2} C_{1}-\delta\right) n$ zeros for the odd positions. Let us consider distributions with fixed even elements. For almost all of them, among the $\geqslant\left(\frac{1}{2} C-\delta\right) n$ positions $2 k+1$ following a one, at least

$$
\left(\frac{1}{2} C-\delta\right)\left(1-\frac{1}{2} C_{1}-\delta\right) n=c_{0} n
$$

will have a zero. There will be $\geqslant c_{0} n$ sequences 1,0 assigned to $2 k, 2 k+1$.

We consider now the set $\mathcal{E}$ of all $3 \times n$ matrices $E$ with $\geqslant C n$ and $\leqslant C_{1} n$ ones, where $0<C<C_{1} \leqslant 1$ are constants. A repeated application of the statement (*) and the above argument establishes

Proposition 6. There are constants $c_{0}$ and $\rho, 0<c_{0}, \rho<1$ so that all but $\rho^{n}|\mathcal{E}|$ matrices $E \in \mathcal{E}$ have at least $c_{0} n$ pairs of columns $2 k, 2 k+1$ which are of type (I) and of type (II) of (3.6).

\section{Proof of the main theorems.}

Proof of Theorem 1. By Proposition 5, most of the matrices $E \in P(m, n)$ contain strips $E_{q}, q=1, \ldots, p$ with some special properties.

Let $\varepsilon>0$ be arbitrary, we take $p$ so large that $2^{-p}<\frac{1}{2} \varepsilon$, and $m \geqslant 3 p$. Next we require that $n$ be so large that $C(p) n^{-1}<\frac{1}{4} \varepsilon$, then Proposition 5 yields that all but $\frac{1}{2} \varepsilon|P(m, n)|$ matrices of the class $P(m, n)$ have the following property. Each strip $E_{q}, q=1, \ldots, p$ of $E$ contains one or more special pairs of columns $2 k, 2 k+1$. This means that $2 k$ is in special position and that the nonzero portion of $E_{q}$ in $2 k$, $2 k+1$ is contained in certain rows $i_{1}<i_{2}<i_{3}$ of $E_{q}$ and is there of type (I) or of type (II) of (3.6). 
Two strips $E_{q}, E_{q}^{\prime}$ are equivalent, $E_{q} \sim E_{q}^{\prime}$, if they have the same set of special pairs $2 k, 2 k+1$, with the same rows $i_{1}<i_{2}<i_{3}$, but perhaps with portions of $E_{q}$, $E_{q}^{\prime}$ contained there of different types (I), (II), and if the strips are identical elsewhere. Two matrices $E, E^{\prime}$ are equivalent if $E_{q} \sim E_{q}^{\prime}, q=1, \ldots, p$.

Let $\bar{P}(m, n)$ be the set of all matrices $E \in P(m, n)$ which satisfy Proposition 5 . We see that this set is invariant under equivalences.

Consider an equivalence class of strips $E_{q}$. We transform a strip $E_{q}$ of this class in an equivalent strip $E_{q}^{\prime}$. Let $2 k, 2 k+1$ be the special pair of $E_{q}$ with smallest possible $k$. Then we replace the portion of $E_{q}$ in these columns and in rows $i_{1}<i_{2}<i_{3}$ into matrix (3.6) of the opposite class. This transformation maps the equivalence class in a one-to-one way onto itself and according to Proposition 4 transforms a nonsingular $E_{q}$ into a singular strip $E_{q}^{\prime}$. Hence at least half of the strips of an equivalence class consists of singular strips. This is true for $q=1, \ldots, p$, and implies that for each equivalence class $\mathcal{E}$ of matrices $E \in \bar{P}(m, n)$ at most $2^{-p}|\mathcal{E}|$ of its matrices are regular. Since $\bar{P}(m, n)$ is a disjoint union of equivalence classes, at most $2^{-p}|\bar{P}(m, n)|<\frac{1}{2} \varepsilon|P(m, n)|$ of its matrices are regular.

Proof of Theorem 2. This is similar. For fixed $m \geqslant 3$ we take $p=[m / 3]$ and divide the $m$ rows of $E$ into $p$ groups of three rows; we neglect the last $\leqslant 2$ rows. For $m=3, p=1$ we cannot use Proposition 5, and special positions $k$ for $E$ need not exist, but we use the singularity Theorem 3 and Proposition 6 instead of Propositions 4, 5.

As a corollary of Theorems 1 and 2 we can offer:

THEOREM 4. Let $\varepsilon>0$ be given, then for $n \geqslant n_{0}(\varepsilon)$ and $m \geqslant 3$, at most $\left(\frac{1}{2}+\varepsilon\right)|P(m, n)|$ matrices of the class $P(m, n)$ are regular.

For $m, n$ that are not necessarily large, we have only

COROLlARY. There exists a number $\delta>0$ for which the probability of singularity of a matrix $E \in P(m, n)$ is $\geqslant \delta$ for each $m \geqslant 3, n \geqslant 2$.

It would be interesting to decide whether the matrices of the class $P(3, n)$ are singular with probability close to 1 for large $n$. However, the singularity theorem given by Proposition 1 is not sufficient for this purpose.

It should be noted that all our theorems (based on Proposition 1) guarantee strong singularity.

\section{BIBLIOGRAPHY}

1. K. Atkinson and A. Sharma, A partial characterization of poised Hermite-Birkhoff interpolation problems, SIAM J. Numer. Anal. 6 (1969), 230-235.

2. G. D. Birkhoff, General mean value and remainder theorems with applications to mechanical differentiation and integration, Trans. Amer. Math. Soc. 7 (1906), 107-136.

3. W. Hoeffding, Probability inequalities for sums of bounded random variables, J. Amer. Statist. Assoc. 58 (1963), 13-30.

4. S. Karlin and J. M. Karon, Poised and non-poised Hermite-Birkhoff interpolations, Indiana Univ. Math. J. 21 (1972), 1131-1170.

5. J. H. B. Kemperman, Moment problems for sampling without replacement, Indag. Math. 35 (1973), $149-188$.

6. G. G. Lorentz, Birkhoff interpolation and the problem of free matrices, J. Approx. Theory 6 (1972), 283-290. 
7. _ "The Birkhoff interpolation problem: New methods and results" in Linear operators and approximation, II edited by P. L. Butzer and B. Sz.-Nagy, ISMN No. 25, Birkhäuser Verlag, Basel, 1974, pp. 481-501.

8. , Coalescence of matrices, regularity and singularity of Birkhoff interpolation problems, $\mathrm{J}$. Approx. Theory 20 (1977), 178-190.

9. G. G. Lorentz and S. D. Riemenschneider, "Recent progress in Birkhoff interpolation" in Approximation theory and functional analysis edited by J. B. Prolla, North-Holland, Amsterdam, 1979, pp. 187-236.

10. , Probabilistic approach to Schoenberg's problem in Birkhoff interpolation theory, Acta Math. Acad. Sci. Hungar. 33 (1979), 127-135.

11. G. G. Lorentz and K. L. Zeller, Birkhoff interpolation, SIAM J. Numer. Anal. 8 (1971), 43-48.

12. R. A. Lorentz, "Interpolation and probability" in Approximation theory, III edited by E. W. Cheney, Academic Press, New York, 1980, pp. 595-600.

13. I. J. Schoenberg, On Hermite-Birkhoff interpolation, J. Math. Anal. Appl. 16 (1966), 538-543.

14. R. J. Serfling, Probability inequalities for the sum in sampling without replacement, Ann. Statist. 2 (1974), 39-48.

Department of Mathematics, University of Texas, Austin, TeXas 78712

Gesellschaft für Mathematik und Datenverarbettung, 5205 St. Augustin, West Germany 